\title{
Dike-Break Induced Flows: a Simplified Model
}

\author{
F. Stilmant ${ }^{1}$, B.J. Dewals ${ }^{1}$, P. Archambeau ${ }^{1}$, S. Roger ${ }^{2}$, S. Erpicum ${ }^{1}$, and M. Pirotton ${ }^{1}$ \\ ${ }^{1}$ Research unit of Hydrology, Applied Hydrodynamics and Hydraulic Constructions (HACH), \\ ArGEnCo Department - MS ${ }^{2} \mathrm{~F}$ - University of Liege (ULg) \\ Chemin des Chevreuils, 1 B52/3 B4000 Liege, Belgium \\ ${ }^{2}$ Institute of Hydraulic Engineering and Water Resources Management (IWW), \\ Aachen University (RWTH) \\ e-mails: \{B.Dewals, Pierre.Archambeau, S.Erpicum, Michel.Pirotton\}@ulg.ac.be
}

\begin{abstract}
A simplified model for the prediction of the steady-state outflow through a breach in an inland dike is presented. It consists in the application of the mass and momentum conservation principles to a macroscopic control volume. A proper definition of the shape of the control volume enables to take into account the main characteristics of the flow and thus to compensate for the extreme simplification of the space discretisation of the model. At the breach, a relation derived from the shallow-water equations is used to determine the directions of the flow. Developments have been guided by numerical simulations and results have been compared to experimental data. Both the precision and the application domain of the simplified model are found satisfactory.
\end{abstract}

\section{Introduction}

Dikes and (mobile) protection walls are essential parts of flood protection measures and considerable damage to people and property can arise from their breaking. A distinction between a dynamic and a static impact is useful to define the focus of a study of dike-break induced flows [1] [2]. As the dynamic impact is related to high flow velocities and water depths within the flood wave initiated by the collapse, it is restricted to the first transient phase of the flow and to the neighbourhood of the breach. On the other hand, the static impact, which is considered in this paper, refers to the slow increase of the water level in the floodplain and affects a wider area and a longer period. Its extent depends on the discharge that leaves the river through the breach, i.e. on a quantity that tends to a steady value for a constant hydrograph, on the supposition that the breach size remains constant.

Over the past few years, a scale model, built at IWW-RWTH Aachen University, has been used to study the transient phase [1] and steady state [2] [3] of dike-break induced flows in a hybrid approach that compared numerical simulations with experimental data. These studies showed that numerical models based on the Shallow-Water Equations (SWE) are able to reproduce the main flow characteristics, yet with some discrepancies. As far as the computation of the steady-state discharge through the breach is concerned, underestimations of experimental data by 5 to $10 \%$ were observed.

Dike breaks are characterized by uncertainties about the flow conditions in the river, the position and the size of the breach. A risk assesment should therefore follow a probabilistic approach and take many scenarios into account, which would result in high computation costs unless a simplified model is used for the computation of the discharge through the breach. Empirical formulae as the one presented in Reference [4] obviously depend on the sites for which they were established. Purpose of this work, instead, has been to develop a physically based model whose validity in different situations can better be comprehended. It is an attempt to reproduce the flow split at a dike breach without any discretisation in space.

The simplified model has been developed in the frame of a scale model (presented in Section 2) and with the help of numerical simulations (Section 3) that have been used to identify useful characteristics of 
the flows (Section 4). The model has been devised as a control volume to which the mass and momentum conservation principles have been applied together with the relevant boundary conditions (Section 5). Developments have mainly consisted in the definition of an appropriate shape for the control volume and in the derivation of a relation giving the flow direction through the breach (Sections 6-7). The numerical implementation of the model has been based on a pseudo-unsteady scheme (Section 8) and the results compare well with experimental data (errors up to $15 \%$ - Section 9).

\section{Experimental data}

Experimental data consist in steady-state discharges measured on a scale model built at IWW [5] [2]. The scale model set-up is presented in Figure 1. It takes into account the boundary conditions of a dike break, yet with a simple geometry, without bottom slope and with low friction so as to focus on the influence of three parameters: the breach width $(30,50$ or $70 \mathrm{~cm})$, the inflow discharge $(100,200$ or 300 $l / s)$ and the initial water depth in the channel $(25$ to $50 \mathrm{~cm}$ - the initial state refers to the steady state with a closed breach). In the following, a generic notation is used to identify the different test configurations with reference to these three parameters. For instance, B70-Q300-h50 refers to a configuration with a $70 \mathrm{~cm}$-wide breach, an inflow discharge of $300 \mathrm{l} / \mathrm{s}$ and an initial water depth of $50 \mathrm{~cm}$. In equations and figures, following notations are used: $B_{c}$ and $B_{b}(m)$ for the channel and breach widths respectively, $Q_{i}$ and $Q_{b}\left(\mathrm{~m}^{3} / \mathrm{s}\right)$ for the discharges in the upstream reach of the channel and through the breach respectively. The whole experimental set-up is comprehensively described in References [5] and [2].

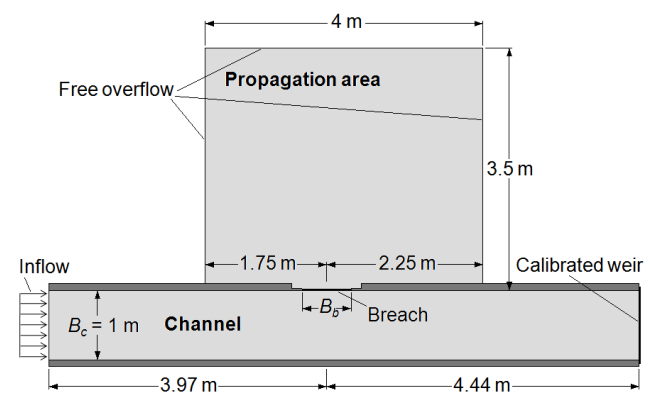

Figure 1: Scale model set-up (adapted from [5])

\section{Numerical simulations}

In Reference [2], numerical simulations were run to reproduce experimental data measured on the scale model described above. One of the software involved in this hybrid approach was the finite volume model WOLF 2D (HACH) which is also used here to expand the set of simulated configurations.

WOLF 2D solves the SWE, which are obtained by depth-averaging the equations of mass and momentum conservation. The derivation of the standard form of the SWE relies on two main assumptions, i.e. low vertical velocity (leading to hydrostatic pressure) and uniform flow velocities over the depth. This mathematical model is solved by a finite volume scheme dealing with multi-block Cartesian grids and limited linear variable reconstruction at cell interfaces to achieve second-order accuracy in space. The computation of fluxes at cell interfaces is based on a flux vector splitting method developed at $\mathrm{HACH}$ [6] [7]. The time integration is performed by means of a dissipative three-step first-order accurate explicit Runge-Kutta algorithm, suitable for the computation of steady flows [2].

In accordance with Reference [2], the Cartesian grid involved $0.02 \mathrm{~m}$ by $0.02 \mathrm{~m}$ cells. However, for configurations with breach widths of 50 and $30 \mathrm{~cm}$, a mesh refinement with $0.01 \mathrm{~m}$ by $0.01 \mathrm{~m}$ cells was used near the breach. The boundary conditions (inflow discharge, weir and free overfall) matched the actual ones described in Figure 1. Notably, the weir equation at the downstream end of the channel required appropriate calibrations [2]. 
In the specific case of the scale model described in Section 2, it was showed that neither bed and wall friction nor turbulence models have a substantial impact on the permanent discharge through the breach [2]. As a consequence and in accordance with the "base simulations" in Reference [2], the present simulations involved a Manning model for bed friction (Manning coefficient of $0.015 \mathrm{~s} / \mathrm{m}^{1 / 3}$ ) but no wall friction and no turbulence model.

\section{Characteristics of the flow near the breach}

The set-up of the scale model ensures that the flow in the channel is subcritical (i.e. Froude number $F<1)$, while it becomes supercritical $(F>1)$ when passing through the breach [2]. Figure 2a sketches the streamline between the two flows that separate at the breach (as deduced from numerical results). It can be seen that the deviated flow undergoes first a lateral contraction in the channel, then a lateral expansion in the propagation area. Maximum contraction is situated at the breach, where the flow regime is critical in the considered configurations [8]. This behaviour is only possible provided that the breach width is not too large compared to the channel width and the percentage of flow deviated through the breach is not too small. The later condition corresponds to a flow regime in the channel characterized by a Froude number not exceeding a given threshold, as discussed below (Section 9).

As mentioned in Reference [2], the computed critical section across the breach takes a distinctive $\mathrm{S}$-shape. Figure $2 \mathrm{~b}$ shows that this shape is influenced by the momentum of the main flow in the channel, i.e. by the Froude number of this flow. At low Froude numbers, the critical section is nearly symmetrical and its basic feature is a curve pointing towards the propagation area. For higher Froude numbers, the momentum of the flow reaching the upstream edge of the breach prevents an immediate deviation, which leads to a S-shape with an important upstream part pointing towards the channel.

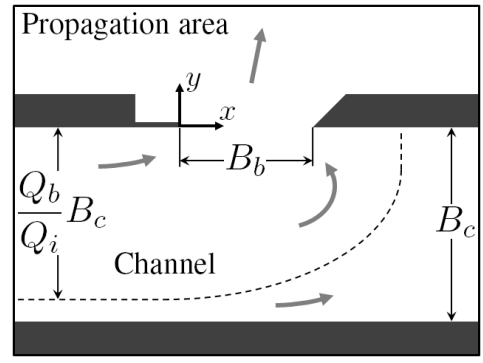

(a)

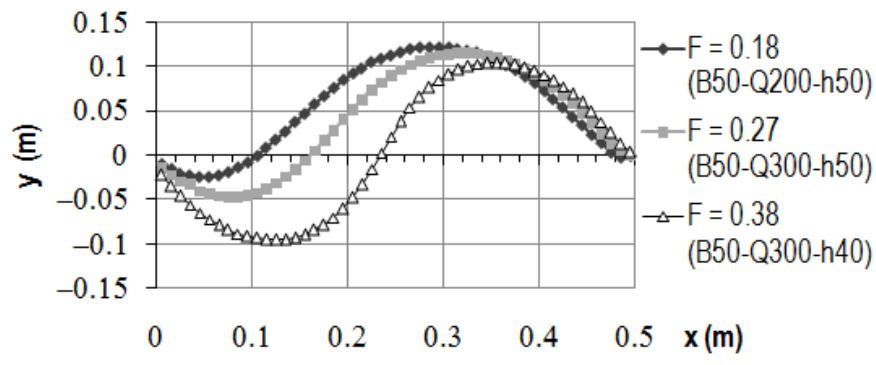

(b)

Figure 2: Characteristics of the flow near the breach - a) Schematic view of the streamline between the two flows that separate at the breach (dotted line) and main flow directions (arrows) - b) Critical sections obtained by interpolation of numerical results in different configurations

\section{Conceptual model}

We suggest a conceptual model based on the application of mass and momentum conservation to a properly defined control volume. When considering only the advective fluxes of the SWE, this approach leads to three equations, in which the unknowns are water depths and discharges along the boundaries of the control volume:

$$
\begin{gathered}
\iint_{A}\left(\partial_{x} \mathbf{f}+\partial_{y} \mathbf{g}\right) d A=0 \quad \Leftrightarrow \quad \oint_{\mathcal{F}+} \mathbf{f} d y-\mathbf{g} d x=0 \\
\mathbf{f}=\left(\begin{array}{c}
q \cos \theta \\
\frac{q^{2}}{h} \cos ^{2} \theta+\frac{q h^{2}}{2} \\
\frac{q^{2}}{h} \sin \theta \cos \theta
\end{array}\right), \quad \mathbf{g}=\left(\begin{array}{c}
q \sin \theta \\
\frac{q^{2}}{h} \sin \theta \cos \theta \\
\frac{q^{2}}{h} \sin ^{2} \theta+\frac{g h^{2}}{2}
\end{array}\right)
\end{gathered}
$$


The coordinate system used throughout this paper is indicated in Figure 2a. In Equation (1), $A$ is the domain of the control volume in the $(x y)$ plane and $\mathcal{F}^{+}$its boundary oriented anticlockwise. The advective fluxes $\mathbf{f}$ and $\mathbf{g}$ are defined by Equation (2), where $h(m)$ is the water depth, $q\left(\mathrm{~m}^{2} / \mathrm{s}\right)$ is the magnitude of the unit discharge and $\theta \in]-\pi ; \pi]$ is the flow direction defined by Equation (3), $q_{x}$ and $q_{y}$ being the unit discharges in $x$ - and $y$-directions respectively:

$$
\left\{\begin{array}{l}
q_{x}=q \cos \theta \\
q_{y}=q \sin \theta
\end{array}\right.
$$

In accordance with the characteristics of the scale model and the conclusions drawn in Reference [2], Equations (1-2) involve no bottom slope and no friction term. For real-life applications of the model, these terms could however be added to the equations, since their effects would be more significant.

The control volume is defined so as to contain the zone in which the flow split occurs, i.e. the zone in the channel where the flow regime is subcritical and the flow parameters are not uniform across the channel width due to the presence of the breach. The use of such a macroscopic control volume requires an appropriate definition of its shape, as shown in following paragraphs.

A flow runs through three of the control volume boundaries, which are used to define the unknowns of the problem (table 1). These unknowns are mean values of water depths and unit discharges along a given boundary. As Equations (1-2) include non linear terms, the use of such mean values is only relevant if the distributions of water depths and unit discharges along the different boundaries are constant. This can be achieved by an adequate definition of the control volume.

Taking into account the three boundary conditions that must be imposed according to the flow regime (table 1), a closed problem is obtained with six equations and six unknowns provided that the shape of the control volume is defined in such a way that flow directions $(\theta)$ through the three boundaries are known. For the "inflow" and "outflow" boundaries, this condition is fulfilled by setting them far enough upstream and downstream from the breach respectively; for the "breach" boundary, specific developments are presented in the following.

\begin{tabular}{|l|l|c|c|}
\hline Boundary & Place & Unknowns & Boundary condition \\
\hline "Inflow" & Across upstream reach & $h_{i}, q_{i}$ & $q_{i}$ imposed \\
"Outflow" & Across downstream reach & $h_{o}, q_{o}$ & $h_{o}=h_{o}\left(q_{o}\right)$ \\
"Breach" & Across breach & $h_{b}, q_{b}$ & $h_{b}=h_{b}\left(q_{b}\right)$ \\
\hline
\end{tabular}

Table 1: Unknowns and boundary conditions

Further developments have therefore consisted in the definition of a proper shape for the control volume so as to simplify the distributions of water depths and unit discharges along the "inflow", "outflow" and "breach" boundaries and to be able to determine the flow directions along them. This shape has been deduced from flow characteristics as disclosed by the results of the $2 \mathrm{D}$ numerical simulations.

\section{Flow through the breach}

The distributions of the flow parameters across the breach section are rather complex and the flow regime is partly supercritical, partly subcritical, as can be deduced from Figure $2 b$. Along this section, the definition of the critical regime, $\mathrm{F}=1$, can thus not be used as a boundary condition linking an average water depth and an average unit discharge. For these reasons, the use of the S-shaped critical section as a boundary of the control volume appears far more appropriate, as the distributions of the unknowns along this curve are simpler and the relation $F=1$ is valid at any point. 


\subsection{Modelling of the critical section}

The use of the critical section as a boundary of the control volume enables number of simplifications, as will be seen in the following subsections, but it requires the definition of a model for this curve. According to the numerical results, flow configurations that differ only by the width $B_{b}$ of the breach display the same non-dimensional critical section shape if coordinates $x$ and $y$ are both scaled with $B_{b}$ (Figure 3a). For the purpose of the computational model presented in this paper, only the main features of this non-dimensional shape need to be modelled and a definition using two parabolae is sufficient. As shown in Figure 3a, this definition requires only three parameters: $\xi_{\text {int }}, \eta_{\min }$ and $\eta_{\max }$, where the non-dimensional coordinates $\xi$ and $\eta$ are defined by

$$
\left\{\begin{array}{l}
\xi=x / B_{b} \\
\eta=y / B_{b}
\end{array}\right.
$$

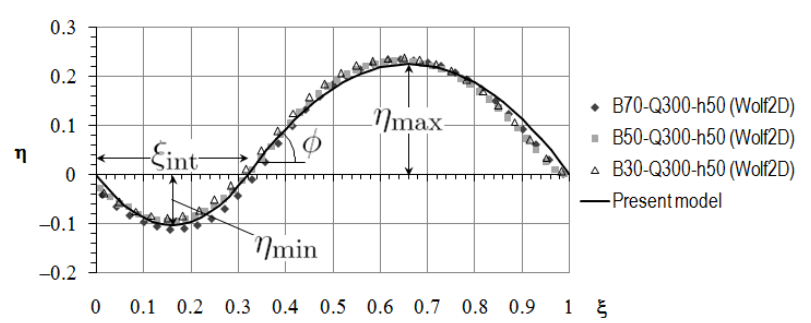

Figure 3: Modelling of the critical section (solid line) and comparison with numerical results (markers)

Parameters $\xi_{\text {int }}, \eta_{\min }$ and $\eta_{\max }$ are not independent. There are two relations between them defining the set of possible shapes of the critical section. In this approach, parameters $\eta_{\min }$ and $\eta_{\max }$ are expressed as concise functions of $\xi_{\text {int }}$ :

$$
\begin{aligned}
\eta_{\min } & =\xi_{\text {int }}^{2} \\
\eta_{\max } & =\frac{1}{4}\left(1-\xi_{\text {int }}^{2}\right)
\end{aligned}
$$

Parameter $\xi_{\text {int }}$ is left as a degree of freedom of the S-shape: it must thus be related to the Froude number of the main flow in the channel, according to section 4 . The Froude number used here is defined as the mean of the Froude numbers at the "inflow" and "outflow" boundaries:

$$
\mathrm{F}_{m}=\frac{1}{2}\left(\mathrm{~F}_{i}+\mathrm{F}_{o}\right)=\frac{1}{2}\left(\frac{q_{i}}{\sqrt{g h_{\imath}^{3}}}+\frac{q_{o}}{\sqrt{g h_{o}^{3}}}\right)
$$

The proposed relation is:

$$
\xi_{\text {int }}=\mathrm{F}_{m}
$$

\subsection{Distribution of water depths and unit discharges along the critical section}

As the S-shape of the critical section is used as a boundary of the control volume, the basics of the dynamic behaviour of the flow across the breach are already included in the model, which therefore makes the best of some simplifications on the water depth and unit discharge distributions along that section. Assuming constant distributions (i.e. constant specific energy along the critical section), the computation of the curvilinear integrals of Equations (1-2) on the critical section $\mathcal{F}_{b}^{+}$gives

- for the continuity equation:

$$
\int_{\mathcal{F}_{b}^{+}} q \cos \theta d y-q \sin \theta d x=\chi_{b} q_{b} B_{b}
$$


- for the momentum equations in $x$ and $y$-directions:

$$
\begin{aligned}
\int_{\mathcal{F}_{b}^{+}}\left(\frac{q^{2}}{h} \cos ^{2} \theta+\frac{g h^{2}}{2}\right) d y-\frac{q^{2}}{h} \sin \theta \cos \theta d x & =\chi_{b x} \frac{q_{b}^{2}}{h_{b}} B_{b} \\
\int_{\mathcal{F}_{b}^{+}} \frac{q^{2}}{h} \sin \theta \cos \theta d y-\left(\frac{q^{2}}{h} \sin ^{2} \theta+\frac{g h^{2}}{2}\right) d x & =\chi_{b y} \frac{q_{b}^{2}}{h_{b}} B_{b}+\frac{g h_{b}^{2}}{2} B_{b}
\end{aligned}
$$

In these equations, coefficients $\chi_{b}, \chi_{b x}$ and $\chi_{b y}$ take into account the curved shape of the boundary (angle $\phi \in]-\pi / 2 ; \pi / 2[$ that gives the direction of the tangent of the curve - Figure 3 ) and the direction of the flow that passes through it (angle $\theta$ ). Using the coordinate $\xi$ defined by Equation (4), leads to:

$$
\begin{aligned}
\chi_{b} & =\int_{0}^{1} \frac{\sin (\theta-\phi)}{\cos \phi} d \xi \\
\chi_{b x} & =\int_{0}^{1} \cos \theta \frac{\sin (\theta-\phi)}{\cos \phi} d \xi \\
\chi_{b y} & =\int_{0}^{1} \sin \theta \frac{\sin (\theta-\phi)}{\cos \phi} d \xi
\end{aligned}
$$

The hypothesis of constant specific energy along the critical section is found to be valid when friction is low: numerical simulations performed with a Manning coefficient of $0.015 \mathrm{~s} / \mathrm{m}^{1 / 3}$ indicate that relative standard deviations of water depths and unit discharges along the critical section are less than $1 \%$. When friction effects do influence the flow split, Expressions (12-14) are less precise. However, as these effects can be taken into account by adding terms in Equations (1-2), the assumption of constant water depths and unit discharges along the critical section could be kept as a working hypothesis.

\subsection{Distribution of flow directions along the critical section}

The assumption of constant specific energy along the critical section provides useful information on the flow parameters in its neighbourhood. The derivation of Bernoulli's specific energy $H$ gives

$$
\vec{\nabla} H=\vec{\nabla}\left(h+\frac{q^{2}}{2 g h^{2}}\right)=\left(1-\mathrm{F}^{2}\right) \vec{\nabla} h+\frac{q}{g h^{2}} \vec{\nabla} q,
$$

which indicates that assuming $\vec{\nabla} H=0$ on the critical section implies $\vec{\nabla} q=0$. The magnitude $\|\vec{\nabla} h\|$ of gradient $\vec{\nabla} h$ remains undefined, but as $q$ is constant along the critical section, it can be deduced from $\mathrm{F}=1$ that the direction of $\vec{\nabla} h$ is locally perpendicular to this curve:

$$
\left\{\begin{array}{l}
\partial_{x} h=\|\vec{\nabla} h\| \cos \left(\phi-\frac{\pi}{2}\right) \\
\partial_{y} h=\|\vec{\nabla} h\| \sin \left(\phi-\frac{\pi}{2}\right)
\end{array}\right.
$$

This information can be used to simplify the SWE on the critical section and thus to establish a relation between the shape of this section and the flow directions. Expressing $\vec{\nabla} q=0$ in the SWE gives:

$$
\begin{aligned}
-\sin \theta \partial_{x} \theta+\cos \theta \partial_{y} \theta & =0 \\
g h \partial_{x} h-\frac{q^{2} \cos \theta}{h^{2}}\left(\cos \theta \partial_{x} h+\sin \theta \partial_{y} h\right)-\frac{q^{2} \sin \theta}{h}\left(\cos \theta \partial_{x} \theta+\sin \theta \partial_{y} \theta\right) & =0 \\
g h \partial_{y} h-\frac{q^{2} \sin \theta}{h^{2}}\left(\cos \theta \partial_{x} h+\sin \theta \partial_{y} h\right)+\frac{q^{2} \cos \theta}{h}\left(\cos \theta \partial_{x} \theta+\sin \theta \partial_{y} \theta\right) & =0
\end{aligned}
$$

Equation (17) indicates that gradient $\vec{\nabla} \theta$ is perpendicular to the flow direction:

$$
\left\{\begin{aligned}
\partial_{x} \theta & =\|\vec{\nabla} \theta\| \cos \theta \\
\partial_{y} \theta & =\|\vec{\nabla} \theta\| \sin \theta
\end{aligned}\right.
$$


Inserting Expressions (16) and (20) in Equations (18-19) finally leads to Equation (21) which gives, in addition to Equation (20), the desired relation between the distributions of $\phi$ and $\theta$. In this equation, $\|\vec{\nabla} h\| / h$ appears to be the only unknown.

$$
\|\vec{\nabla} \theta\|=\frac{\|\vec{\nabla} h\|}{h} \cos (\theta-\phi)
$$

Assuming a constant $\|\vec{\nabla} h\|$ along the critical section enables the construction of different distributions of angle $\theta$, as illustrated in Figure 4a. For this purpose, Equations (20-21) have been projected in the local direction of the critical section, which results in Equation (22), formulated based on the non-dimensional coordinate $\xi$. Notation $K$ has been used for $\|\vec{\nabla} h\| B_{b} / h$.

$$
d_{\xi} \theta=\|\vec{\nabla} h\| \frac{B_{b}}{h} \frac{\cos ^{2}(\theta-\phi)}{\cos \phi}=K \frac{\cos ^{2}(\theta-\phi)}{\cos \phi}
$$

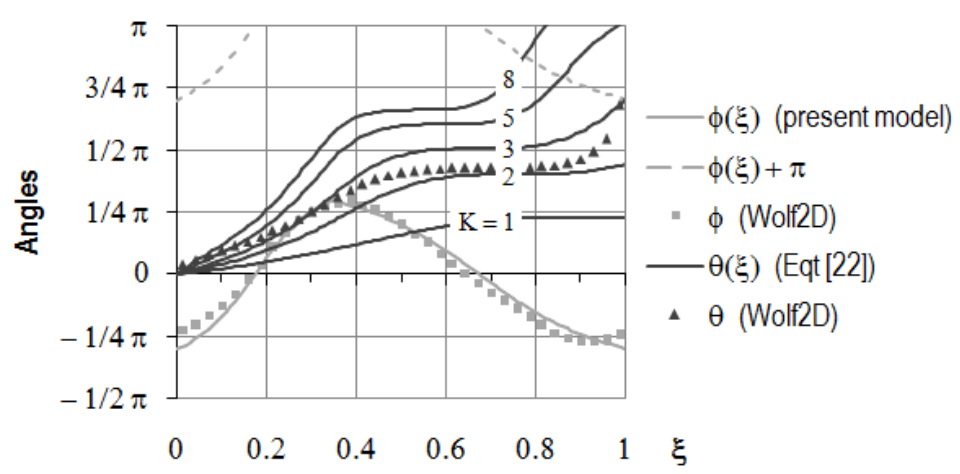

(a)

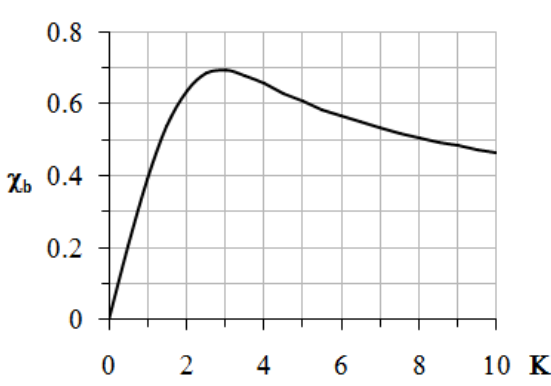

(b)

Figure 4: Parametrical study of the influence of parameter $K$ on - a) the distribution of angle $\theta$ along the critical section - b) coefficient $\chi_{b}$ (configuration B70-Q300-h50)

Figure 4a shows that the different $\theta$-profiles which can be computed thanks to Equation (22) mostly involve some points where Equation (23) is not verified:

$$
\phi(\xi) \leq \theta(\xi) \leq \phi(\xi)+\pi
$$

The effectiveness of the critical section in evacuating a discharge $Q_{b}$ for a given head $H_{b}$ is reduced by these points at which flow doesn't leave the channel but (re-)enters it. Equation (24), derived from (9) and the definition of critical regime, shows that this effectiveness is indicated by coefficient $\chi_{b}$ :

$$
Q_{b}=\chi_{b} q_{b} B_{b}=\frac{2}{3} \frac{\chi_{b}}{\sqrt{3}} \sqrt{2 g} H_{b}^{3 / 2} B_{b}
$$

For a given critical section, it therefore seems rational to choose the distribution of $\theta$ that gives maximum $\chi_{b}$ (Figure $4 \mathrm{~b}$ ) for the computation of coefficients $\chi_{b}, \chi_{b x}$ and $\chi_{b y}$.

\section{Flow in the channel}

Purpose of this section is to define the distances from the breach at which the "inflow" and "outflow" boundaries should be placed in order to ensure uniform water depths and unit discharges distributions across the channel and flow directions parallel to the $x$-axis. These distances obviously depend on the width $B_{c}$.

However, placing these sections far enough from the breach is not enough: their position should make the definition of coefficients expressing the uneven distribution of pressure fluxes along the wall 
boundaries of the control volume as simple as possible. Such coefficients, named $\chi_{k}$, appear when deriving Equation (1). They are defined by

$$
\int_{B_{k}} \frac{g h^{2}}{2} d x=\chi_{k} \frac{g h_{\mathrm{ref}, k}^{2}}{2} B_{k}
$$

where subscript $k$ refers to a given wall boundary, $B_{k}$ is the length of the boundary and $h_{\mathrm{ref}, k}^{2}$ can be $h_{i}^{2}, h_{o}^{2}$ or a combination of both. Figure 5 shows that the pressure fluxes on the right wall of the channel are almost symmetric with respect to abscissa $x_{\text {int }}=\xi_{\text {int }} B_{b}$. Making use of this result and giving the control volume a length $L=2 B_{c}+2 x_{\text {int }}$ leads to the simple and constant coefficients given in Table 2 .

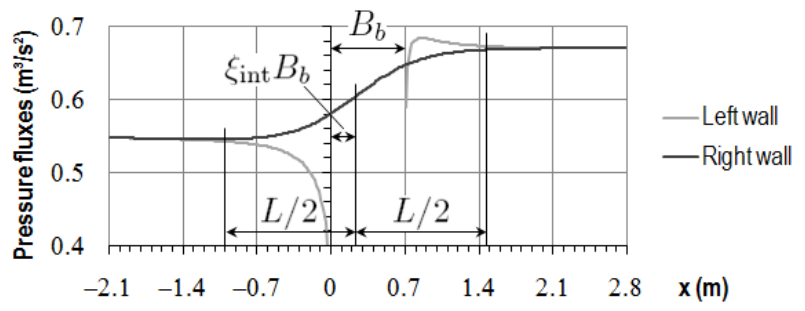

Figure 5: Distribution of $g h^{2} / 2$ along the walls of the channel (configuration B70-Q300-h50)

\begin{tabular}{|l|c|c|c|c|}
\hline Wall & Subscript $k$ & $h_{\text {ref }, k}^{2}$ & $B_{k}$ & $\chi_{k}$ \\
\hline Right & $r$ & $\left(h_{i}^{2}+h_{o}^{2}\right) / 2$ & $2 B_{c}+2 x_{\text {int }}$ & 1,00 \\
Left - upstream & $l u$ & $h_{i}^{2}$ & $B_{c}$ & 0,95 \\
Left - downstream & $l d$ & $h_{o}^{2}$ & $B_{c}+2 x_{\mathrm{int}}-B_{b}$ & 1,00 \\
\hline
\end{tabular}

Table 2: Definition of coefficients $\chi_{k}$

\section{System of equations and numerical scheme}

Writing out in full Equation (1) for the control volume defined in Sections 6 and 7 leads to:

$$
\begin{aligned}
\partial_{t} h_{i} & =\frac{1}{A}\left[q_{i} B_{c}-q_{o} B_{c}-\chi_{b} q_{b} B_{b}\right] \\
\partial_{t} q_{o} & =\frac{1}{A}\left[\left(\frac{q_{i}^{2}}{h_{i}}+\frac{g h_{i}^{2}}{2}\right) B_{c}-\left(\frac{q_{o}^{2}}{h_{o}}+\frac{g h_{o}^{2}}{2}\right) B_{c}-\chi_{b x} \frac{q_{b}^{2}}{h_{b}} B_{b}\right] \\
\partial_{t} q_{b} & =\frac{1}{A}\left[\left(\chi_{r} \frac{B_{r}}{2}-\chi_{l u} B_{l u}\right) \frac{g h_{i}^{2}}{2}+\left(\chi_{r} \frac{B_{r}}{2}-\chi_{l d} B_{l d}\right) \frac{g h_{o}^{2}}{2}-\chi_{b y} \frac{q_{b}^{2}}{h_{b}} B_{b}-\frac{g h_{b}^{2}}{2} B_{b}\right]
\end{aligned}
$$

Left-hand sides of these equations, that are zero for the steady state flows of interest, have been introduced for the sake of computation. According to the SWE, these three terms should respectively be the integrals of $\partial_{t} h, \partial_{t} q_{x}$ and $\partial_{t} q_{y}$ over the domain $A \approx 2 B_{c}^{2}$ of the control volume. In a pseudo-unsteady approach computing the steady-state solution starting from an arbitrary initial condition, these integrals can be supposed to be proportional to the time-derivatives of three of the six unknowns of the problem.

The system of Equations (26-28), expressing mass and momentum conservation, must be closed by following boundary conditions, which are algebraic relations to be solved at each pseudo-time step to give the values of the unknowns $q_{i}, h_{o}$ and $h_{b}$ :

$$
\begin{aligned}
q_{i} & =\frac{Q_{i}}{B_{c}} \\
q_{o} & =\frac{2}{3}\left(0.657+0.084 \frac{h_{o}-h_{w}}{h_{w}}\right) \sqrt{2 g}\left(h_{o}-h_{w}\right)^{3 / 2} \\
q_{b} & =\sqrt{g h_{b}^{3}}
\end{aligned}
$$


In this case, $Q_{i}$ is a given parameter of the problem. Equation (30) is the weir relation calibrated for the weir at the end of the channel as given in Reference [5] ( $h_{w}$ is the crest heigth). A more rigorous relation would imply a 1D computation of water depths along the downstream reach of the channel but this proved to be unnecessary in the present case (low friction and short reach) and Equation (30) is kept here for clarity.

The suggested time-integration algorithm is a three-step first-order accurate Runge-Kutta scheme. A Courant-Friedrichs-Lewy number is introduced in Equations (26-28) for stability reasons. It can generally be set to unity, except when $q_{o}$ approaches zero.

\section{Results}

In Figure 6a, the ratio $Q_{b} / Q_{i}$ is plotted against the initial Froude number $\mathrm{F}_{0}$, which can be obtained thanks to $q_{i}$ and Equation (30). The simplified model shows good agreement with WOLF 2D simulations, from which it derives: the absolute difference between results is less or equal to 5\% (the corresponding relative difference is less or equal to 15\%). The consistency with the experimental data is less (absolute error up to $15 \%$ and relative error up to $17 \%$ ), which is to be put down to the inherent hypotheses of the SWE according to References [2] and [3].

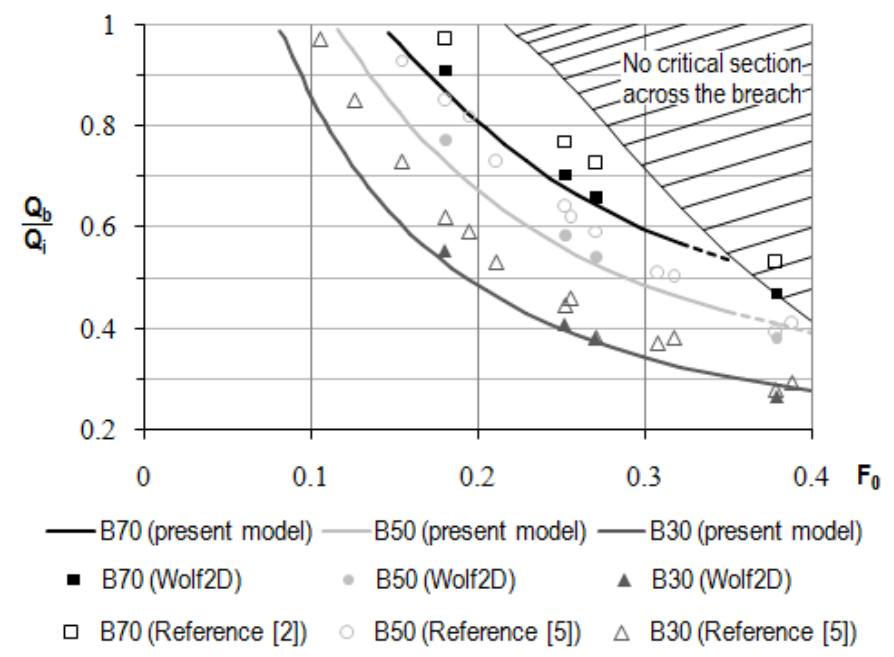

(a)

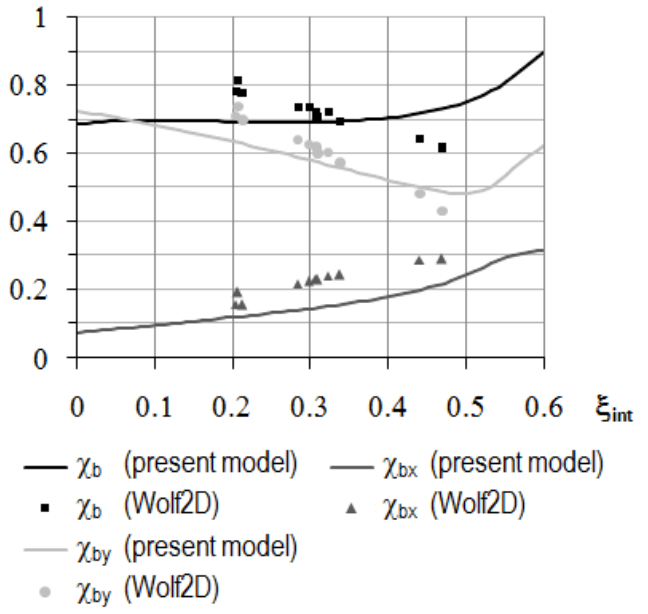

(b)

Figure 6: Comparison of results given by the simplified model with experimental data and/or results given by WOLF 2D: - a) $Q_{b} / Q_{i}$ ratio as a function of the initial Froude number $\mathrm{F}_{0}$ for different breach widths - b) $\chi_{b}, \chi_{b x}$ and $\chi_{b y}$ coefficients as functions of $\xi_{\text {int }}$

The validity of the model is linked to the contraction effect of the deviated flow at the breach (see Section 4): when this effect is not reproduced by the model (i.e. $q_{b} \leq q_{i}$ ), the corresponding solution is not valid. Figure $6 \mathrm{~b}$ compares $\chi_{b}, \chi_{b x}$ and $\chi_{b y}$ coefficients as defined in Section 6 with their values given by the interpolation of WOLF $2 \mathrm{D}$ results. At low $\xi_{\text {int }}$ (i.e. low $\mathrm{F}_{m}$ ), these three coefficients are underestimated by the present model, while they are overestimated for $\xi_{\text {int }}>0.4-0.5$. The later behaviour results in a reduced flow contraction effect, which reduces the application domain of the simplified model.

Solid lines giving the results of the model in Figure 6a have been replaced by dotted lines for configurations with $\mathrm{F}_{m}>0.5$, i.e. when $\chi_{b}, \chi_{b x}$ and $\chi_{b y}$ coefficients are significantly overestimated. Figure $6 \mathrm{a}$ also shows the domain where the application of the simplified model is not relevant because the hypothesis of a critical section across the breach is not valid (hatched area). This domain has been computed thanks to the simplified model, which means that its extend is slightly overestimated as the condition $\mathrm{F}_{m}<0.5$ is not fulfilled in this zone. For configurations with $B_{b} / B_{c} \leq 1$, the application of the present 
model is possible for initial Froude numbers lower than 0.3. For lower $B_{b} / B_{c}$ ratios, flows with higher initial Froude numbers can be computed.

\section{Conclusion}

A simplified model, based on a macroscopic control volume, has been developed for the prediction of the steady-state outflow through a dike breach. The absence of space discretisation has required the definition of a proper shape for the control volume so as to take advantage of the main characteristics of the flow, as disclosed by 2D numerical simulations, and of simplifications of the Shallow-Water Equations on the critical section.

In view of its simplification, this model has been found to compare well with numerical simulations and experimental data. Its application domain has also been investigated focusing on its main hypothesis, i.e. a critical section crossing the breach, and appears to be large enough to cover many fluvial conditions.

The scale model set-up has been well-suited for the study of the advective terms of the simplified model. For real-life applications however, friction and bottom slope terms should be added in the system of equations, as their effects would be more significant. Similarly, it is worth verifying the behaviour of the simplified model in configurations with larger $B_{c} / B_{b}$ and $B_{b} / h$ ratios.

\section{References}

[1] S. Roger, E. Büsse, and J. Köngeter. Dike-break induced flood wave propagation. In P. Gourbesville and al., editors, $7^{\text {th }}$ International Conference on Hydroinformatics, Nice, France, 2006.

[2] S. Roger, B.J. Dewals, S. Erpicum, D. Schwanenberg, H. Schüttrumpf, J. Köngeter, and M. Pirotton. Experimental and numerical investigations of dike-break induced flows. Journal of Hydraulic Research, 47:349-359, 2009.

[3] S. Roger, B.J. Dewals, S. Erpicum, D. Schwanenberg, P. Archambeau, J. Köngeter, M. Pirotton, and H. Schüttrumpf. Hybrid modelling of dike-break induced flows. In A. Dittrich and al., editors, $5^{\text {th }}$ International Conference on Fluvial Hydraulics (Riverflow), Braunschweig, Germany, 2010.

[4] P. Kamrath, M. Disse, M. Hammer, and J. Köngeter. Assessment of discharge through a dike breach and simulation of flood wave propagation. Natural Hazards, 38:63-78, 2006.

[5] S. Briechle. Die fächenhafte Ausbreitung der Flutwelle nach Versagen von Hochwasserschutzeinrichtungen an Fließgewässern. PhD thesis, Rheinisch-Westfälische Technische Hochschule Aachen, 2006. In German.

[6] B.J. Dewals, S.A. Kantoush, S. Erpicum, M. Pirotton, and A.J. Schleiss. Experimental and numerical analysis of flow instabilities in rectangular shallow basins. Environmental Fluid Mechanics, 8:31-54, 2008.

[7] S. Erpicum, B.J. Dewals, P. Archambeau, and M. Pirotton. Dam-break flow computation based on an efficient flux-vector splitting. Journal of Computational \& Applied Mathematics, 234:2143-2151, 2010.

[8] W.H. Hager. Critical flow condition in open channel hydraulics. Acta Mechanica, 54:157-179, 1985. 